\title{
Disruptive Teaching Methodology for STEM Education
}

\author{
Ajay Krishnan $M^{1 *}$, Amol Deshpande ${ }^{2}$ \\ ${ }^{1}$ COMSOL Multiphysics Pvt Ltd, Bengaluru \\ ${ }^{2}$ Department of Chemical Engineering, BITS Pilani K K Birla Goa Campus, Goa \\ Iajaykrishnan.muralidharan@comsol.com \\ 2 amoldeshpande@goa.bits-pilani.ac.in
}

\begin{abstract}
:
Innovative teaching methodologies are of prime interest in the current evolving education sector to improve the student learning experiences. This paper talks about an attempt on implementing a new disruptive teaching methodology based on COMSOL Applications as a teaching aid for the Computational Fluid Dynamics Laboratory (based on Transport Phenomena course) at BITS Pilani Goa Campus. This study showcased the fact that such teaching methods aid students to gain a deeper understanding of a concept and assist them in retaining the knowledge rather than just memorizing formulas. Implementation and evaluation of this new teaching methodology are discussed.
\end{abstract}

Keywords: Active Learning, Simulation tools, Teaching aids.

\section{Introduction:}

India has been one of the frontrunners in the teaching methodology that dates back to the $10^{\text {th }}$ century with the Gurukool teaching system. With the evolution of the education sector, especially Science, Technology, Engineering, and Mathematics (STEM) education, the focus has been shifted to imbibing an immersive learning experience for the students. The shift in the teaching pedagogy is deemed to be highly crucial as the demands from STEM education have changed from gaining mere theoretical knowledge to developing skills such as critical thinking, reasoning, and innovation which help students shape their professional careers. Therefore, over the years, teaching methodology has evolved from a chalk-talk way of teaching to PowerPoint presentations and finally to the current Active learning era. Active learning involves and aids students in understanding the natural laws and promotes a conceptual understanding as opposed to comprehending through traditional lectures.

Bonwell et al. mentioned that active learning methodology not only involves students in doing things but also in thinking about what they are doing[1]. Felder et al. showed [2] that for a chemical engineering course, students who were taught by an active and cooperative learning approach outperformed their traditionally-taught peers, in terms of both knowledge retention and graduation rate. Richard Hake [3] showed reviewed data from over 6000 physics students in 62 introductory physics courses and found that students in classes that utilized active learning techniques improved 25 percentage points, achieving an average gain of $48 \%$ on a standard test of conceptual physics knowledge compared to an increase of $23 \%$ for students in traditional, lecture-based courses. Owing to such promising outcomes, many institutions are finding ways to implement such active learning methodologies in their classrooms. Physical laboratories are traditionally used as an active learning tool to give students an insight into the concept being taught in the classroom, thereby promoting conceptual learning [4]. Although they look promising, it is indeed difficult for many institutions to establish physical laboratories, that come with a hefty cost and further maintenance issues. With the advent of technology and the ongoing pandemic situation, the usage of simulation tools as a teaching aid in the classrooms to create an active learning environment has 
gained momentum. Deshpande et al. [5] mentioned that the application of simulation tools in engineering education had helped students immensely and changed the educational landscape remarkably. It is possible that by re-organizing or adapting the learning material presented to students, an the opportunity to correlate the concepts taught in the classroom about unobservable phenomena such as chemical reactions, fluid flow, thermodynamics or electricity [9-12], and link the unobservable processes to governing equations [13]. This paper details about an attempt on utilising a new disruptive teaching methodology based on COMSOL Applications to promote active learning amongst students at the Birla Institute of Technology and Science Pilani, Goa campus, India for a Computational Fluid Dynamics laboratory (practical section of Transport Phenomena course).

\section{Implementation:}

Transport phenomena is one of the foundation courses in the Chemical Engineering curriculum. Owing to its multidisciplinary nature, it is often a challenging course to teach as well. Understanding the momentum, heat, and mass transfer are the three pillars of the Transport Phenomena course. This study is intended to maximize the efficiency of the learning process of undergraduate and graduate level Chemical engineering students by introducing complex concepts in the Transport Phenomena course as CFD simulation - laboratory section. In this section, students are asked to model, simulate, predict, understand and validate the transport behaviour (i.e. fluid flow, heat transfer and mass transfer behaviour) of standard chemical engineering systems using COMSOL Multiphysics ${ }^{\circledR}$ and COMSOL Applications. COMSOL Multiphysics ${ }^{\circledR}$ consists of various predefined core physics interfaces for environment that promotes knowledge retention can be created. Several literature reports say that $[6,7,8]$ simulation tools as a teaching aid help better knowledge retention and promote inquiry-based learning. In addition, students have

different engineering disciplines, offering flexibility to generate Multiphysics numerical models. By leveraging the inbuilt Application Builder functionality, an intuitive and specific user interface for the Transport phenomena (CFD lab section) course has been created. COMSOL Applications are built based on the BITS Pilani, Goa Chemical Engineering curriculum. Some of these case studies are discussed below. The core benefit of using COMSOL Applications is that the implementation cost is drastically minimized while still providing an opportunity to visualize unobservable phenomena. COMSOL Application for flow past a cylinder is shown in Figure 1. The interface was built with the mindset to make the learning more intuitive and exciting for the students. These applications solve the necessary partial differential equations in the background, where unnecessary details are hidden from the user via a simplified user interface (UI). As a result, students don't have to be simulation experts to use these applications. They can change the predetermined parameters by adjusting the application interface, and visualize results based on their choices. In this example, Navier Stokes equations are being solved in the background, and students can visualize the velocity and pressure profiles by changing the cylinder dimensions. In addition, the application also accounts for Laminar vs Turbulent flows based on the inlet velocity and also provides an option to model a variety of fluids with varying density and viscosity. 


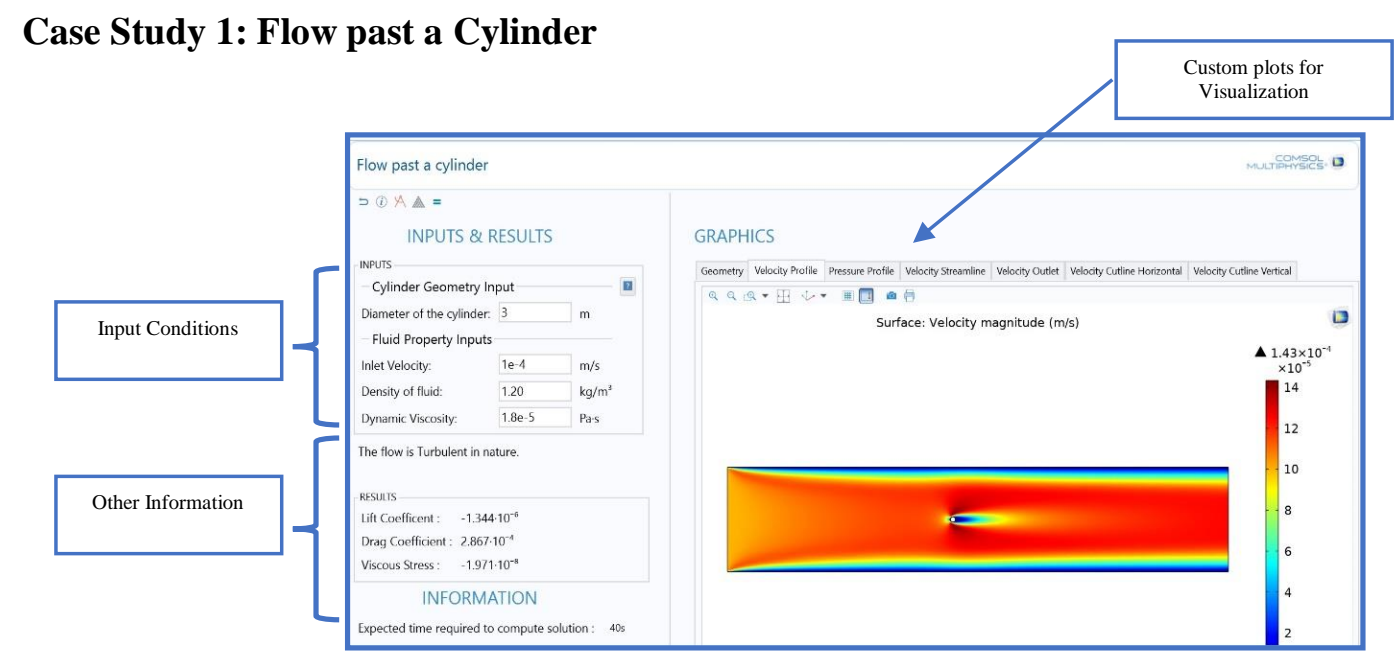

Figure 1: COMSOL Application ${ }^{\mathrm{TM}}$ on Flow Past a Cylinder

It is essential to point out here that the application triggers "What If" learning amongst the students when they try out different combinations provided in the UI. COMSOL Application for Heat Exchanger is shown in Figure 2. Heat exchangers are common in many industries as they are typically used to cool the fluids. This application is designed in such a way that it establishes the industrial importance of the equipment and provides a compelling reason for the students to understand the framework of Heat Exchangers. Moreover, the application provides an opportunity for the students to understand different kinds of Heat Exchangers (Current \& Co-Current) based on the flow configuration. This application gives an insight into designing Heat exchangers whilst optimizing the temperature profiles based on provided inputs.

Deployment:To make things easier for the students and to get the classes going during this pandemic situation, COMSOL Applications can be deployed using COMSOL Server. COMSOL Server enables students to access and run these applications through web browsers using computers, tablets, or even their smartphones, thereby making these applications more accessible both in the classroom and at home. In addition to deploying these applicatios over server, these COMSOL Applications can also be converted into standalone executable (.exe) files using the COMSOL Compiler for distribution. With a click of a button, students can download the runtime files needed to run the exe files and use it throughout their semester without any hassle.

\section{Observations:}

Transport phenomena have been in the Chemical Engineering curriculum for ages. It is a common notion among professors that teaching transport phenomena courses is tricky as they have a lot of mathematics in the subject. Such notions are also agreed upon by students, as translating Fourier's law or Navier-Stokes equations or Heat Conduction equations into a mental picture of either a flow pattern or temperature profile is not an easy task. COMSOL Multiphysics ${ }^{\circledR}$ and Applications that were designed and implemented at BITS Pilani, Goa campus enabled undergraduate students (a class of 70 students) and graduate students (10 students) to develop an interest in the subject through interactive visualizations provided in the applications. It is also observed that some concepts which students find difficult to understand in the conventional classroom, can be effectively presented here in relatively less time. It is essential to highlight that this pilot 
project encouraged various students to go and test their hypotheses by changing the parameters given in the application, which they might have been hesitant to test out otherwise in a physical laboratory. By utilizing such an innovative, disruptive teaching methodology, students as well as faculties found these solutions more intuitive, rather than relying on memorizing formulas to solve a problem. It is important to hightlight that many students have developed further interest in the subject because of the change in teaching methodology and number of students working in the projects related to the field of Transport Phenomena have increased by two times in the span of a year. The pilot project proved that by providing such an interactive interface for students and supplementing it with passive lecture videos, students were able to develop a deeper understanding of the actual phenomenon through inquiry-based learnin

\section{Case}

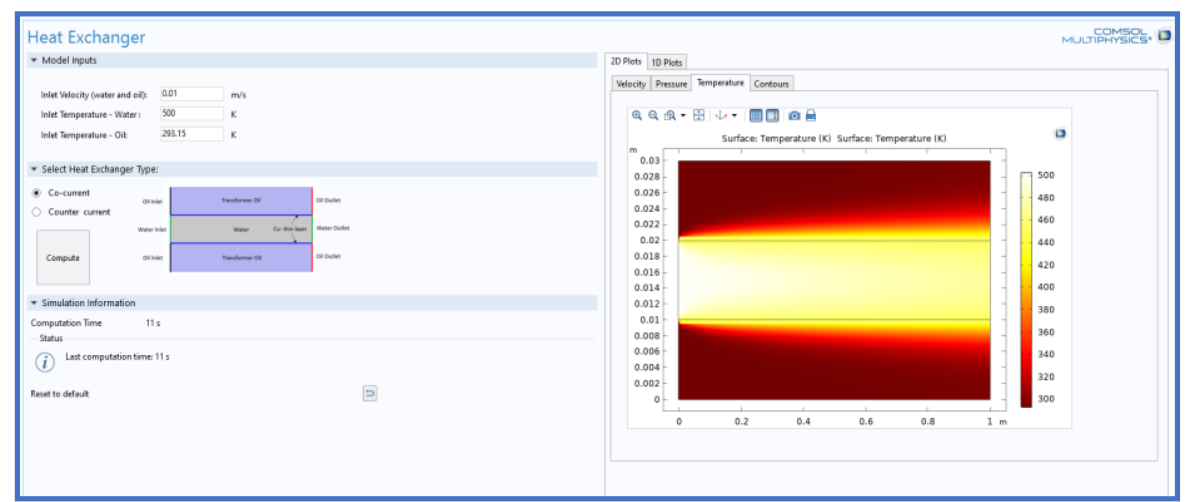

Study 2: Heat Exchanger

Figure 2: COMSOL Application ${ }^{\mathrm{TM}}$ on Heat Exchanger

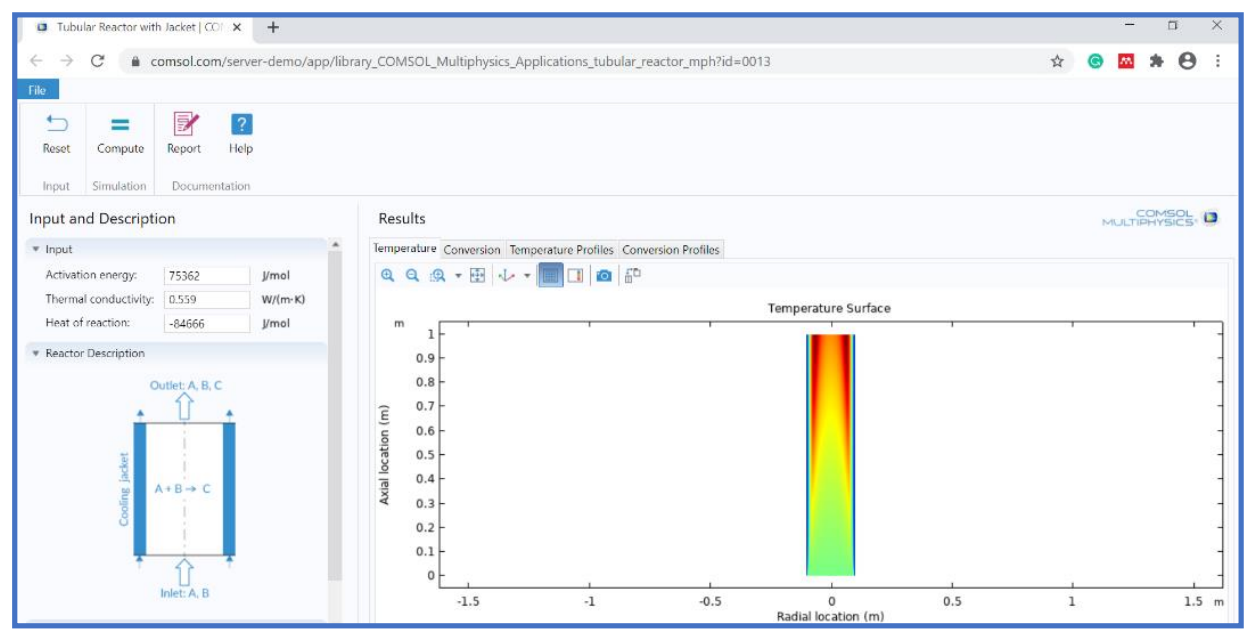

Figure 3: COMSOL Application ${ }^{\mathrm{TM}}$ on Tubular Reactor accessed via a web browser

\section{Summary}

Technology cannot replace teachers. However, with the advancement in educational technology, it is possible to achieve a better learning experience with the use of such teaching aids. Utilizing COMSOL
Multiphysics $^{\circledR}$ and COMSOL Applications at BITS Pilani, Goa campus, helped both undergraduate and graduate Chemical engineering students to visualize unobservable phenomena like fluid flow velocity, temperature profiles, pressure profiles, and 
correlate with the governing equations taught in the classroom. With the help of COMSOL Server, the applications can be deployed and accessed over a web browser to enable a higher-order learning experience for the students.

\section{References}

[1] C. Bonwell, J. Eison, Active Learning: Creating Excitement in the Classroom. 1991 ASHE-ERIC Higher Education Reports., 1991.

[2] R.M. Felder, A Longitudinal Study of Engineering Student Performance and Retention. IV. Instructional Methods, J. Eng. Educ. 84 (1995) 361-367.

[3] R.R. Hake, Interactive-engagement versus traditional methods: A sixthousand-student survey of mechanics test data for introductory physics courses, Am. J. Phys. 66 (1998) 64-74.

[4] National Research Council, America's Lab Report: Investigations in High School Science, S. R. Singer, M. L. Hilton, H. A. Schweingruber, Eds. (National Academy Press, Washington, DC, 2006).

[5] A.A. Deshpande, S.H. Huang, Simulation games in engineering education: A state-of-the-art review, Comput. Appl. Eng. Educ. 19 (2011) 399-410.

[6] P. McClean, B. Saini-Eidukat, D. Schwert, B. Slator, and A. White, In: J. A. Chamber (Ed.), Virtual worlds in large enrollment science classes significantly improve authentic learning, Selected Papers from the 12th International Conference on College Teaching and Learning, Center for the Advancement of Teaching and Learning, Jacksonville, FL, 2001, pp 111-118.
[7] K. Squire, Video games in education, Int J Intell Games Simul 2 (2003), 49-62.

[8] K. Squire, M. Barnett, J. M. Grant, and T. Higginbotham, Electromagnetism supercharged! Learning physics with digital simulation games, in Proceedings of the 2004 International Conference of the Learning Sciences, UCLA Press, LA, 2004.

[9] Z. C. Zacharia, C. P. Constantinou, Comparing the influence of physical and virtual manipulatives in the context of the Physics by Inquiry curriculum: The case of undergraduate students' conceptual understanding of heat and temperature, Am. J. Phys. 76, 425 (2008).

[10] T. Jaakkola, S. Nurmi, K. Veermans, A comparison of students' conceptual understanding of electric circuits in simulation only and simulation-laboratory contexts, J. Res. Sci. Teach. 48, 71 (2011).

[11] Z. H. Zhang, M. C. Linn, Can generating representations enhance learning with dynamic visualizations?, J. Res. Sci. Teach. 48, 1177 (2011).

[12] L. Deslauriers, C. E. Wieman, Learning and retention of quantum concepts with different teaching methods, Phys. Rev. Spec. Topics Phys. Educ. Res. 7, 010101 (2011).

[13] K. W. McElhaney, M. C. Linn, Investigations of a complex, realistic task: Intentional, unsystematic, and exhaustive experimenters, J. Res. Sci. Teach. 48, 745 (2011). 